\title{
Study on Demand Response of Residential Power Customer
}

\author{
Xiu Cao',2, Haiyong Jiang1,2, Lei Huang3, Xueping Wang1,2, Xuqi Zhang1,2 \\ ${ }^{1}$ School of Computer Science, Fudan University, Shanghai, China \\ ${ }^{2}$ Engineering Research Center of Cyber Security Auditing and Monitoring, Ministry of Education, Shanghai, \\ China \\ ${ }^{3}$ Xinneng Kaibo Industrial Co. Ltd., Shanghai, China \\ Email:xiucao@fudan.edu.cn, 12210240090@fudan.edu.cn, $13901738202 @ 139 . c o m$, \\ wangxp@fudan.edu.cn,15210240105@fudan.edu.cn
}

Received 9 March 2016; accepted 16 July 2016; published 19 July 2016

\begin{abstract}
In order to optimize the ladder-pricing scheme in Shanghai, we present a multi-objective optimization model (MOOM). To build this model, first we use price elasticity theory; divide the ladder pricing into peak electricity bill and valley electricity bill in the time dimension to model the single-user demand response. Second based on the single-user demand response model, combined with the overall users' electricity distribution density function, we build an all-users demand response model. The proposed model has two objectives: minimize energy consumption and maximize residents' satisfaction. Simulation results confirm that the proposed model can optimize the ladder-pricing scheme.
\end{abstract}

\section{Keywords}

Demand Response, Ladder Pricing, Price Elasticity, Ladder Pricing Optimization

\section{Introduction}

Over a longer period of time, as a result of a unitary low price has been implemented in China which had a problem of cross-subsidization [1] [2], there was a serious contradiction between industrial (commercial) users and residential users. To alleviate this problem, in recent years, our country carried out a series of bill reforms and has implemented TOU, ladder-pricing which is also known as cumulative price ladder and so on. The ladder pricing not only can ease the cross-subsidization problem off [3], but also can inhibit the residents from wasting electricity. Since 2012, the practice of ladder pricing, the existing ladder program requires constant optimization [4] to adjust to changes in different factors, for example, residential customer income, energy, environment, etc.

Ladder pricing is based on Ramsey rule [5]. Taking advantage of Ramsey rule is a way to make the ladder pricing scheme [6]. According to the analysis of factors which affect the electric price, such as residents' affordability, residential electricity demand, the cost of electricity and so on, the authors [7] has proposed tier quantity optimal model and the tier range optimal model. Rank-Sum Ratio method and round-robin algorithm are applied in [8] to find the best tiered electric quantity setting. The authors [9] first discuss the difference in elasticity 
of consumers based on Stone-Geary Function and then develop an optimization model to determine the optimal tiered levels. The author [10] takes advantage of Ramsey pricing principle and elasticity matrix of elasticity demand and builds a joint optimization model of residential time-of-user block electricity rate.

The discussions and analysis in this paper are based on the above literature. In this paper, we construct a single-user demand response model for users with different stalls in ladder pricing. Then combined the overall users' electricity distribution density function, an all-users demand response model is established. Finally, we propose a multi-objective optimization model whose objectives are to minimize energy consumption and maximize residents' satisfaction.

The rest of this paper is organized as follows. We introduce elasticity in Section 2. The single-user demand response model and all-users demand response model are formulated in Section 3. The multi-objective optimization model is presented in Section 4. In Section 5, simulation results are shown.

\section{Elasticity}

In microeconomics, the elastic theory is mainly used for researching the measurement of how an economic variable is to change in another [11].

\subsection{Price Elasticity of Demand}

Price elasticity of demand is one of elasticity, commonly referred to as the price elasticity.

Price elasticity of demand primarily used to represent a period of time, the extent of the relative change in the demand for commodity reactions with the relative changes in the price of the commodity itself. Price elasticity of demand usually represented by the following formula:

$$
\varepsilon=\frac{\frac{\Delta Q}{Q}}{\frac{\Delta P}{P}}=\frac{\Delta Q}{\Delta P} \cdot \frac{P}{Q}
$$

where $\varepsilon$ is the price elasticity of demand coefficient, $\Delta Q$ is change in quantity demanded, $\Delta P$ is change in price, $Q$ is quantity demanded, $P$ is price.

\subsection{Cross-Price Elastic}

Under normal circumstances, the demand for a commodity is not only concerned with its own price, but also related to the price of similar products. Also in the electricity market, as in the TOU conditions, the energy in the time dimension of peak, valley, flat can be seen as three different goods, user demand for electricity usually depends not only on the flat period price, but also related to peak and valley time price [12]. In order to characterize this relationship, introduced the cross elasticity of demand [11].

Cross elasticity of demand mathematical expression is as follows:

$$
\varepsilon_{x y}=\frac{\frac{\Delta Q_{X}}{Q_{X}}}{\frac{\Delta P_{Y}}{P_{Y}}}=\frac{\Delta Q_{X}}{\Delta P_{Y}} \cdot \frac{P_{Y}}{Q_{X}}
$$

where $\varepsilon_{X Y}$ is the cross-price elasticity of demand coefficient, $\Delta Q_{X}$ is change in X's quantity demanded, $\Delta P_{Y}$ is change in Y's price, $Q_{X}$ is X's quantity demanded, $P_{y}$ is Y's price.

\subsection{Elastic Matrix for Electricity Price}

Defines price elasticity matrix:

$$
E_{i}=\left[\begin{array}{ll}
\varepsilon_{p p} & \varepsilon_{p v} \\
\varepsilon_{v p} & \varepsilon_{v v}
\end{array}\right]
$$

where $E_{i}$ is the price elasticity matrix of the $i$-tier user, $\varepsilon_{p p}$ is the Price elasticity of demand of the peak pe- 
riod, $\varepsilon_{v v}$ is the Price elasticity of demand of valley period, $\varepsilon_{p v}$ is the cross-price elasticity of demand between peak period and valley period, $\varepsilon_{v p}$ is the cross-price elasticity of demand between valley period and peak period.

\section{Demand Response}

\subsection{Single-User Demand Response}

Changes in user requirement matrix can be formulated as follows:

$$
\left[\begin{array}{c}
\Delta q_{p} \\
\Delta q_{v}
\end{array}\right]=\left[\begin{array}{c}
\sum_{i=1}^{3}\left(\Delta q_{p p, i}+\Delta q_{p v, i}\right) \\
\sum_{i=1}^{3}\left(\Delta q_{v v, i}+\Delta q_{v p, i}\right)
\end{array}\right]
$$

where $\Delta q_{p}$ is change in electric energy demanded at peak period, $\Delta q_{v}$ is change in electric energy demanded at valley period, $\Delta q_{p p, i}$ is change in electric energy demanded at peak period of the $i$-tier user caused by change in peak price, $\Delta q_{p v, i}$ is change in electric energy demanded at peak period of the $i$-tier user caused by change in valley price, $\Delta q_{v v, i}$ is change in electric energy demanded at valley period of the $i$-tier user caused by change in valley price, $\Delta q_{v p, i}$ is change in electric energy demanded at valley period of the $i$-tier user caused by change in peak price.

Take (1)-(3) into (4), we can get:

$$
\left[\begin{array}{c}
\Delta q_{p} \\
\Delta q_{v}
\end{array}\right]=E_{i}\left[\begin{array}{l}
\sum_{i=1}^{3} q_{p, i} \cdot \frac{\Delta p_{p, i}}{p_{p}} \\
\sum_{i=1}^{3} q_{v, i} \cdot \frac{\Delta p_{v, i}}{p_{v}}
\end{array}\right]
$$

where $q_{p, i}$ is the $i$-tier peak electric energy consumption, $q_{v, i}$ is the $i$-tier valley electric energy consumption, $p_{p}$ is the first-tier peak price, $\left(\Delta p_{p, i}+p_{p}\right)$ is the new $i$-tier peek price, $p_{v}$ is the first-tier valley price, $\left(\Delta p_{v, i}+p_{v}\right)$ is the new $i$-tier valley price.

Ladder pricing in Shanghai is divided into three levels by user electric energy consumption, assume the lowest level consumption in the $\left(0, x_{1}\right)$ range, the middle level consumption in the $\left(x_{1}, x_{2}\right)$ range, the highest level in the $\left(x_{2}, \infty\right)$ range. For each user in three levels, we have

$$
\begin{gathered}
q_{1}=q_{p, 1}+q_{v, 1}+\varepsilon_{p p} \cdot \frac{q_{p, 1}}{p_{p}} \cdot \Delta p_{p, 1}+\varepsilon_{p v} \cdot \frac{q_{p, 1}}{p_{v}} \cdot \Delta p_{v, 1}+\varepsilon_{v v} \cdot \frac{q_{v, 1}}{p_{v}} \cdot \Delta p_{v, 1}+\varepsilon_{v p} \cdot \frac{q_{v, 1}}{p_{p}} \cdot \Delta p_{p, 1} \\
q_{p, 1}+q_{v, 1} \leq x_{1} ; \\
q_{2}=\sum_{i=1}^{2} q_{p, i}+q_{v, i}+\sum_{i=1}^{2}\left(\varepsilon_{p p} \cdot \frac{q_{p, i}}{p_{p}} \cdot \Delta p_{p, i}+\varepsilon_{p v} \cdot \frac{q_{p, i}}{p_{v}} \cdot \Delta p_{v, i}+\varepsilon_{v v} \cdot \frac{q_{v, i}}{p_{v}} \cdot \Delta p_{v, i}+\varepsilon_{v p} \cdot \frac{q_{v, i}}{p_{p}} \cdot \Delta p_{p, i}\right) \\
q_{p, 1}+q_{v, 1}=x_{1}, 0<q_{p, 2}+q_{v, 2} \leq x_{2}-x_{1} ; \\
q_{3}=\sum_{i=1}^{3} q_{p, i}+q_{v, i}+\sum_{i=1}^{3}\left(\varepsilon_{p p} \cdot \frac{q_{p, i}}{p_{p}} \cdot \Delta p_{p, i}+\varepsilon_{p v} \cdot \frac{q_{p, i}}{p_{v}} \cdot \Delta p_{v, i}+\varepsilon_{v v} \cdot \frac{q_{v, i}}{p_{v}} \cdot \Delta p_{v, i}+\varepsilon_{v p} \cdot \frac{q_{v, i}}{p_{p}} \cdot \Delta p_{p, i}\right) \\
q_{p, 1}+q_{v, 1}=x_{1}, q_{p, 2}+q_{v, 2}=x_{2}-x_{1}, q_{p, 3}+q_{v, 3}>0 ;
\end{gathered}
$$

where $q_{i}$ is the i-tier user energy demand after responding to change in price.

From (6), we can see the first-tier user only responds to the first-tier pricing changes, the second-tier user needs to responds to the first-tier and second tier pricing changes and the third-tier user needs to responds to all the three tiers pricing changes.

In this paper, we suppose that all the users do not shift from one tier to another after they respond to the pricing changes.

The pricing changes, and the user has regulated their demand, the electricity bill can be obtained as:

$$
c_{1}=\left(\Delta q_{p p, 1}+\Delta q_{p v, 1}+q_{p, 1}\right) \cdot\left(p_{p}+\Delta p_{p, 1}\right)+\left(\Delta q_{v v, 1}+\Delta q_{v p, 1}+q_{v, 1}\right) \cdot\left(p_{v}+\Delta p_{v, 1}\right),
$$




$$
\begin{aligned}
& c_{2}=\sum_{i=1}^{2}\left(\Delta q_{p p, i}+\Delta q_{p v, i}+q_{p, i}\right) \cdot\left(p_{p}+\Delta p_{p, i}\right)+\left(\Delta q_{v v, i}+\Delta q_{v p, i}+q_{v, i}\right) \cdot\left(p_{v}+\Delta p_{v, i}\right), \\
& c_{3}=\sum_{i=1}^{3}\left(\Delta q_{p p, i}+\Delta q_{p v, i}+q_{p, i}\right) \cdot\left(p_{p}+\Delta p_{p, i}\right)+\left(\Delta q_{v v, i}+\Delta q_{v p, i}+q_{v, i}\right) \cdot\left(p_{v}+\Delta p_{v, i}\right)
\end{aligned}
$$

where $c_{i}$ is the $i$-tier user electricity bill after responding to change in price

\subsection{All-Users Demand Response}

We have formulated single-user demand response, now the question is: How to get the all-users demand response? To answer this question, we applied the overall users electricity distribution density function. Define $f(x)$ as the peak electric energy consumption $f(y)$ as the valley electric energy consumption and:

$$
\begin{aligned}
& \forall 0<q<+\infty, F(q)=\int_{0}^{q} f(x) d x, \\
& \forall 0<q<+\infty, F(q)=\int_{0}^{q} f(y) d y
\end{aligned}
$$

Given $f(x), f(y)$, the electric energy consumption and electricity bill can be derived as:

$$
\begin{gathered}
Q^{*}=\sum_{i=1}^{i=3} U \cdot \gamma_{i} \cdot\left(\int_{0}^{\infty} f\left(q_{i, p}\right) q_{i, p}^{*} d q_{i, p}+\int_{0}^{\infty} f\left(q_{i, v}\right) q_{i, v}^{*} d q_{i, v}\right) \\
C^{*}=\sum_{i=1}^{i=3} U \cdot \gamma_{i} \cdot\left(p_{i, p}^{*} \cdot \int_{0}^{\infty} f\left(q_{i, p}\right) q_{i, p}^{*} d q_{i, p}+p_{i, v}^{*} \cdot \int_{0}^{\infty} f\left(q_{i, v}\right) q_{i, v}^{*} d q_{i, v}\right)
\end{gathered}
$$

where $Q^{*}$ is electricity energy demand of all the users after responding to change in price, $C^{*}$ is electricity bill of all the users after responding to change in price, $U$ is the total number of users, $\gamma_{i}$ the proportion of $i$-tier users, $f\left(q_{i, p}\right)$ is the peak energy consumption density function of $i$-tier users, $f\left(q_{i, v}\right)$ is the valley energy consumption density function of $i$-tier users, $q_{i, p}^{*}$ is peak electricity energy demand of $i$-tier users after responding change in the price, $q_{i, v}^{*}$ is valley electricity energy demand of $i$-tier users after responding change in the price, $p_{i, p}^{*}$ is $i$-tier new peak price, $p_{i, v}^{*}$ is $i$-tier new valley price.

\section{Multi-Objective Optimization Model}

\subsection{Satisfaction}

The satisfaction of the residential customer can be modeled as:

$$
\begin{gathered}
\theta=1-\frac{C^{*}-C}{C} \\
\text { s.t. }\left\{\begin{array}{c}
0 \leq C^{*}-C \leq \eta C \\
0 \leq \eta \leq 1
\end{array}\right.
\end{gathered}
$$

where $\theta$ is the satisfaction of residents, $\eta$ is upper bound coefficient of the growth of electricity gross proceeds.

Clearly, the lower $C^{*}$ is the higher satisfaction the customer will get. If $C^{*}$ is equal to $C$, satisfaction will approach the upper bound 1 . If $C^{*}$ is twice than $C$, satisfaction will be close to 0 .

\subsection{Energy Consumption}

Implementation ladder pricing policy, an important goal is to guider resident users to reduce electricity consumption, improve power efficiency, to increase the utilization of electric power system [13]. In this paper, we name $\rho$ as electricity coefficient to represent the ration of the amount of total electricity after response to that before response. The electricity coefficient is as:

$$
\rho=\left(Q_{p}^{*}+Q_{v}^{*}\right) /\left(Q_{p}+Q_{v}\right)
$$

where $Q_{p}{ }^{*}$ is peak electricity energy demand of all the users after responding to change in price, $Q_{v}{ }^{*}$ is valley electricity energy demand of all the users after responding to change in price, $Q_{p}$ is peak electricity energy demand of all the users, $Q_{v}$ is valley electricity energy demand of all the users. 
On considering only price influence of factors on residential electricity consumption condition, when the electricity price increases, the overall residential users' electricity consumption should show a negative trend. It is reasonable to assume that we always have the following constraint:

$$
Q_{p}^{*}+Q_{v}^{*}-Q_{p}-Q_{v} \leq 0
$$

\subsection{Optimization Model}

So far, we are ready to formulate the ladder pricing optimization problem as the following multi-objective optimization problem:

$$
\begin{aligned}
& \left\{\begin{array}{l}
\min \rho \\
\max \theta
\end{array}\right. \\
& \text { s.t. }\left\{\begin{array}{c}
\rho=\frac{Q_{p}^{*}+Q_{v}^{*}}{Q_{p}+Q_{v}}, \\
\theta=1-\frac{C^{*}-C}{C},(2) \\
0 \leq C^{*}-C \leq \eta C, \\
0 \leq \eta \leq 1,4 \\
0.05 \leq \Delta p_{p, 2}<\Delta p_{p, 3}, \\
0.3 \leq \Delta p_{p, 3}, 6 \\
\Delta p_{v, 2}=\frac{\Delta p_{p, 2}}{2}, \quad 7 \\
\Delta p_{v, 3}=\frac{\Delta p_{p, 3}}{2},
\end{array}\right.
\end{aligned}
$$

From (14),

guarantee electricity bill growth after user response within a certain range.

tional Development and Reform Commission.

that valley electricity bill is half of the peak electricity bill.

In the above optimization problem, the two objectives conflict with each other, we cannot find the optimal solution to meet these two objectives. So in this article, we will use genetic algorithm to find the Pareto set of this model.

\section{Simulation Result}

\subsection{Data}

According to the guidance of NDRC, this paper supposes that the first-tier electricity price will not change, the second-tier peak electricity price relative to the first-tier peak will increase $\Delta p_{p, 2} \mathrm{RMB} / \mathrm{kWh}$, the second-tier valley electricity price will increase $\Delta p_{p, 2} / 2 \mathrm{RMB} / \mathrm{kWh}$, the third-tier peak electricity price relative to first-tier electricity peak price will increase $\Delta p_{p, 3} \mathrm{RMB} / \mathrm{kWh}$, and the third-tier valley electricity price will increase $\Delta p_{p, 3} / 2$ $\mathrm{RMB} / \mathrm{kWh}$. The electricity energy consumption standard for each tier in Shanghai will remain the same.

The experimental data are actual consumption data of 1487 residents in an area of Shanghai in 2012. From these data, statistical results show that:

1) There are 1242 residents belongs to first-tier user. Average electricity consumption per month is $167 \mathrm{kWh}$. Ratio between peak and valley is 0.692:0.308;

2) There are 217 residents belongs to first-tier user. Average electricity consumption per month is $293 \mathrm{kWh}$. Ratio between peak and valley is 0.691:0.309;

3) There are 28 residents belongs to first-tier user. Average electricity consumption per month is $380 \mathrm{kWh}$. Ratio between peak and valley is 0.694:0.306.

Taking the limited electricity historical data into account, the data of electric power elasticity matrix refer- 
ences that in [10].

Table 1 figures out that the first-tier resident and the third-tier resident has a small price elastic, lack of elastic, to the contrary the second-tier resident has a large price elastic, and is sensitive to electrovalence.

\subsection{Density Distribution Function}

By using $\mathrm{R}$ to test the distribution of peak and valley power consumption for each tier customer, the result shows that they are all belongs to lognormal distribution. The lognormal distribution is as follows:

$$
f(x ; \mu, \sigma)=\frac{1}{x \sigma \sqrt{2 p i}} \mathrm{e}^{-(\ln x-\mu)^{2} / 2 \sigma^{2}}
$$

The parameters of these density functions can be worked out through the history power data of 1473 residents, which are shown in Table 2.

\subsection{Result and Analysis}

We use Matlab to solve the multi-objective optimization model (13) by genetic algorithm. Finally the result of this optimization problem is shown as follows:

From the result, we can see when $\Delta p_{p, 1}$ is 0.077 and $\Delta p_{p, 2}$ is 1.462 , energy consumption is lowest but the residents satisfaction is highest. On the opposite, when $\Delta p_{p, 2}$ is 0.2998 and $\Delta p_{p, 3}$ is 0.4023 , energy consumption is highest but the resident satisfaction is lowest.

If we take the current ladder pricing policy of Shanghai into (7), the result shows the 1478 residents can conserve $502.378 \mathrm{kWh}$ energy the whole year, the extra electricity bill is $4561.142 \mathrm{RMB}$ and the resident satisfaction is 0.997 .

Now if the ladder pricing decision makers want to save more energy relatively, when $\Delta p_{p, 2}$ is 0.102 and $\Delta p_{p, 3}$ is 0.823 , the saving energy will improve from $502.378 \mathrm{kWh}$ to $745.999 \mathrm{kWh}$. On the other hand, someday they want to improve the resident satisfaction relatively, from the Figure 1, when $\Delta p_{p, 2}$ is 0.054 and $\Delta p_{p, 3}$ is 0.501 , the resident satisfaction can improve from 0.997 to 0.998 .

Table 1. Price elastic and cross-price elastic.

\begin{tabular}{cccc}
\hline User & Period & Price Elastic $\varepsilon_{p p}\left(\varepsilon_{v v}\right)$ & Cross-Price Elastic $\varepsilon_{p v}\left(\varepsilon_{v p}\right)$ \\
\hline \multirow{2}{*}{ First-tier Resident } & Peak & -0.0654 & 0.0138 \\
& Valley & -0.0606 & 0.0207 \\
Second-tier Resident & Peak & -0.0903 & 0.0294 \\
& Valley & -0.0849 & 0.0396 \\
Third-tier Resident & Peak & -0.0513 & 0.0159 \\
& Valley & -0.0447 & 0.0234 \\
\hline
\end{tabular}

Table 2. The parameters of the density function.

\begin{tabular}{ccc}
\hline Density Function & $\mu$ & $\sigma$ \\
\hline$f\left(q_{1, p}\right)$ & 7.150205 & 0.4095554 \\
$f\left(q_{1, v}\right)$ & 6.30344 & 0.5020718 \\
$f\left(q_{2, p}\right)$ & 7.758021 & 0.2832774 \\
$f\left(q_{2, v}\right)$ & 6.920627 & 0.3824835 \\
$f\left(q_{3, p}\right)$ & 8.025475 & 0.2658047 \\
$f\left(q_{3, v}\right)$ & 7.163866 & 0.4158537 \\
\hline
\end{tabular}




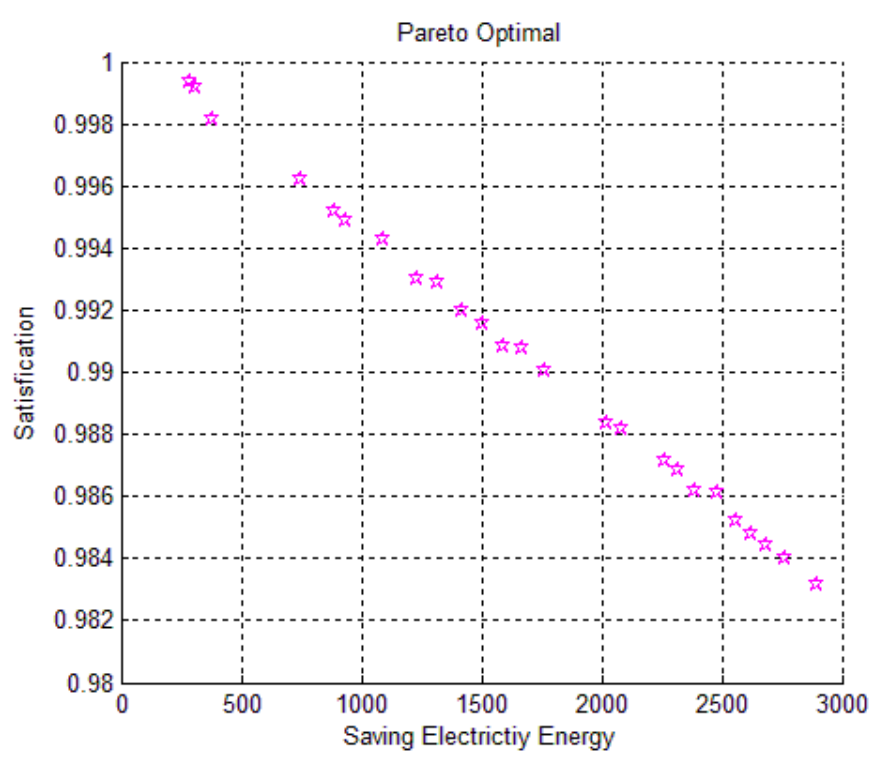

Figure 1. Pareto optimal solutions.

\section{Conclusions and Future Work}

In this paper, we have first analyzed the single-user demand response and all-users demand response. Based on these and combined with the density function of energy consumption, we have proposed a multi-objective optimization model. Through the optimization model, the design makers can formulate different ladder pricing scheme for various purpose in different period.

Here, we have just focused on optimal the price of ladder pricing. A higher research on optimal both the price and the tier range of ladder pricing may be done in the future.

\section{References}

[1] Zhang, L.Z. (2010) Discussion of Residents Stepped Tariff System Increments. Price Theory and Practice, 2, 9-10.

[2] Zhu, C.Z. (2010) The Ladder Pricing Is the Ladder of Tariff Reform. China Power Enterprise Management, No. 11.

[3] Gao, Y. (2012) Research Timesharing Ladder Pricing Based on TOU and Ladder Pricing. Journal of Sichuan University of Science \& Engineering: Natural Science Edition, 25.

[4] Li, C.R. and Yu, J.M. (2010) Korean Residents of the Ladder Pricing Experience and Enlightenment. Electric Power Technologic Economics, No. 7, 56-61.

[5] Lin, B.Q. (2010) Controversy of Ladder Pricing. China Power Enterprise Management, No. 27,

[6] Brown, S.J. (1986) Sibley David Sumner. The Theory of Public Utility Pricing. Cambridge University Press, Cambridge. http://dx.doi.org/10.1017/CBO9780511666773

[7] Wang, W.L. and Liu, J.C.(2012) Influence Factors of Ladder Pricing and Analysis of Optimization Model. Foreign Investment in China, No. 18.

[8] Zhu, K.D. and Song, Y.H. (2011) Tan Zhognfu. Residents Ladder Pricing Design Optimization Model. East China Electric Power, No. 6.

[9] Li, Y., Luo, Q. and Song, Y.Q. (2012) Study on Tiered Level Determination of TOU \& Tiered Pricing for Residential Electricity Based on Demand Response. Power Protection and Control System, No. 18.

[10] Huang, H.T. (2012) A Joint Optimization Model of Residential Time-of-Use Block Electricity Rate. Grid Technology, No. 10.

[11] Economics. http://en.wikipedia.org/wiki/Elasticity_

[12] Qin, Z.F., Yue, S.M. and Yu, Y.X. (2004) End Retail Electricity Market Electricity Price Elasticity Matrix. Automation of Electric Power Systems, No. 5.

[13] Liu, Y., Tan, Z.F. and Qi, J.X. (2005) TOU Pricing Design Optimization Model. China Management Science, No. 5. 